Original Contribution

\title{
BILATERAL ULTRASOUND-GUIDED ABDOMINAL PERIPHERAL BLOCK IN TAP PLANE, TAP - BLOCK
}

\author{
M. Sekulovski ${ }^{1,2 *}$, B. Simonska ${ }^{1}$, G. Mutafov ${ }^{1}$, V. Alexandrov ${ }^{1,2}$, L. Spassov ${ }^{1,2}$ \\ ${ }^{1}$ Clinic of Anesthesiology and Intensive Care Unit, UH "Lozenetz" Sofia, Bulgaria \\ ${ }^{2}$ Faculty of Medicine, SU “St. Kliment Ohridski”, Sofia, Bulgaria
}

\begin{abstract}
INTRODUCTION: Bilateral ultrasound-guided peripheral block (TAP - block) in the plane between the inner oblique abdominal muscle and the transversal abdominal muscle - TAP plane, is a regional anesthesia technique by infiltration of a local anesthetic, provides analgesia for operations involving the anterior abdominal wall. The analgesic effectiveness of the block decreases the consumption of opioid analgesics and non-steroidal anti-inflammatory drugs.

AIM: In this study, we evaluated the intraoperative analgesic efficacy of bilateral TAP - block and the consumption of opioid analgesics in patients undergoing bilateral laparoscopic inguinal hernia repair. METHODS: The study was conducted with 35 patients, who were randomized into two groups. In the control group (group I), there are patients who received general anesthesia (GA), and experimental group (group II), were patients who have received general anesthesia and a bilateral tap block (GA + TAP).

RESULTS: Patients with TAP-block (group II) have significantly lower fentanyl consumption compared to group I.

CONCLUSION: Multimodal approach for the simultaneous administration of general anesthesia with a TAP block provides effective intraoperative analgesia and significantly reduces the perioperative consumption of opioid analgesics.
\end{abstract}

Key words: local anesthesia, multimodal anesthesia, ultrasound transversus abdominis plane block, opioid, pain

\section{INTRODUCTION}

Inguinal hernia repair is one of the most common surgeries. Between 300 and 600 operative methods, modifications and techniques for the surgical treatment of inguinal hernia have been described in the literature. However, the laparoscopic technique for the treatment of inguinal hernias has been admitted to reduce postoperative pain, to provide early recovery and less hospital stay for patients (Takebayashi et al, 2015). Patients under bilateral laparoscopic inguinal repair reported varying severity of pain. Good pain control is important and we have been using multimodal anesthesiology approaches,

\footnotetext{
*Correspondence to: $M$. Sekulovski, $U H$

"Lozenetz" - Clinic of anesthesiology and intensive care unit, Sofia, Bulgaria, Faculty of medicine, SU

"St. Kliment Ohridski", Sofia, Bulgaria

1407, Sofia, 1 Kozyak str.,

metodijabt@hotmail.com, Tel.+359896714 321
}

regional techniques and drug combinations in recent years [1].

The ultrasound-guided abdominal peripheral block in the TAP plane (the neuromuscular plane between the inner oblique abdominal muscle and the transversus abdominis muscle), also known as TAP block, is a regional anesthesiology technique that blocks somatic nerves (Th7-Th11) [2].

The TAP block was introduced into anesthesiology practice in 2001 by Rafi, who used the lumbar triangle of Petit [3]. Hebbard et al. in 2007 described for the first time the use of ultrasound on a TAP block, which made the method much more efficient [4].

Subsequent clinical trials are leading to the increasing use of the TAP block as part of a multimodal approach for anesthesia for postoperative analgesia in various surgical 
procedures: caesarean sections $[5,6]$, open and laparoscopic hernia repair [7, 8], appendectomy [9], colon resection [10], prostatectomy [11] and other surgical procedures involving the anterior abdominal wall [12].

The majority of studies in this area confirm that the TAP block provides good postoperative analgesia, but there are few studies evaluating its intraoperative analgesic efficacy.

The purpose of this study is to evaluate intraoperative analgesic efficacy, opioid analgesic consumption, and pain assessment by visual-analogue scale during extubation and displacement, using a TAP block in combination with general anesthesia, in patients undergoing bilateral laparoscopic inguinal repair.

\section{MATERIAL AND METHODS}

The study was performed with 35 patients (men) between 24 and 55 years old, with a Classification of ASA (American Society of Anesthesiologists) class I and II at University Hospital "Lozenetz", Sofia, Bulgaria, between January-December, 2017. All patients signed informed consent after explaining to them the manipulation in detail. The patients were categorized into two groups. In group I $(\mathrm{n}=17)$, patients were administered with general anesthesia $(\mathrm{OA})$ and in group II $(\mathrm{n}=18)$ general anesthesia and tap block (OA-TAP). Exclusion criteria included a patient: less than 18 years of age, ASA class III-IV, allergy to local anesthetic, coagulation disorders and patients with body mass index (BMI)> $35 \mathrm{~kg} / \mathrm{m} 2$. Laparoscopic hernia repair was performed by the same surgical team.

Demographic characteristics of the patients patients' age, body mass index, and ASA classification are presented in Table $\mathbf{1 .}$

Table 1. Demographic characteristics of the patients studied. Values are presented as mean \pm standard deviation (Mean $\pm S D$ ).

\begin{tabular}{|c|c|c|c|}
\hline \multicolumn{3}{|c|}{ Demographic characteristic } & \multirow[b]{2}{*}{$P$ - value } \\
\hline & $\begin{array}{c}\text { Group I } \\
\text { (GA) }\end{array}$ & $\begin{array}{c}\text { Group II (GA + } \\
\text { TAP) }\end{array}$ & \\
\hline Age, years & $32,24 \pm 9,90$ & $35,06 \pm 8,02$ & $\mathrm{P}=0,18$ \\
\hline Body weight, kg & $1,80 \pm 0,08$ & $1,81 \pm 0,06$ & $P=0,34$ \\
\hline Body height, $m$ & $90,94 \pm 9,07$ & $88,72 \pm 8,90$ & $\mathrm{P}=0,24$ \\
\hline BMI & $28,06 \pm 2,76$ & $27 \pm 1,93$ & $\mathrm{P}=0,10$ \\
\hline$A S A I$ & $82 \%, n=14$ & $78 \% \quad(n=13)$ & \multirow[b]{2}{*}{$P=0,37$} \\
\hline$A S A I I$ & $18 \%, n=3$ & $22 \% \quad(\mathrm{n}=5)$ & \\
\hline
\end{tabular}

Multimodal anesthesia was administered: Propofol, Lysthenon, Fentanyl and Sevoflurane. Premedication of patients was performed with midazolam at a dose of 0.05 $\mathrm{mg} / \mathrm{kg}$, per os, 40 minutes before the patient was transported to the operating room by the anesthesiology team. Induction of general anesthesia in all patients was the same Propofol-Lipuro $1 \%$ at a dose of $2 \mathrm{mg} / \mathrm{kg}$; Fentanyl at a dose of $0.5-1 \mathrm{mcg} / \mathrm{kg}$ and muscle relaxant - Lysthenon $1 \%$ at a dose of 1 $\mathrm{mg} / \mathrm{kg}$. After 90 seconds of mask ventilation, endotracheal intubation was performed. For maintenance of anesthesia in both groups of patients: GA - general anesthesia without TAP block (control group) and GA+TAP - general anesthesia using TAP block (experimental group), we used inhalation anesthetic
Sevoflurane 0.7-1.2 MAK (minimum alveolar concentration), muscle relaxant - Tracrium and bolus doses, if necessary with opioid analgesic - Fentanyl at a dose of $0.5 \mathrm{mcg} / \mathrm{kg}$.

Perioperative monitoring included: heart rate, non-invasive blood pressure, electrocardiogram, pulse oximetry ( $\mathrm{SpO} 2)$, capnography, and bispectral index monitoring (BIS). Monitoring for inadequate analgesia included various symptoms such as sweating, tearing, tachycardia (heart rate above 80 beats / $\min$ ), hypertension (> $20 \%$ of baseline blood pressure).

After induction of anesthesia and intubation, in the patients from the GA+TAP group bilateral TAP - block was performed under ultrasound control. For the implementation of the TAP - 
block we used portable ultrasound machine Sonoscape S2, linear transducer 5-11 MHz's. Patients were positioned in the supine position. The transducer was placed transversely along the median axillary line in the middle of the space between the iliac crest and the costal margin to views skin, subcutaneous tissue, fat and the three abdominal wall muscles (external and internal oblique abdominal muscle, and transverse abdominal muscle), including the peritoneum (Figure 1).

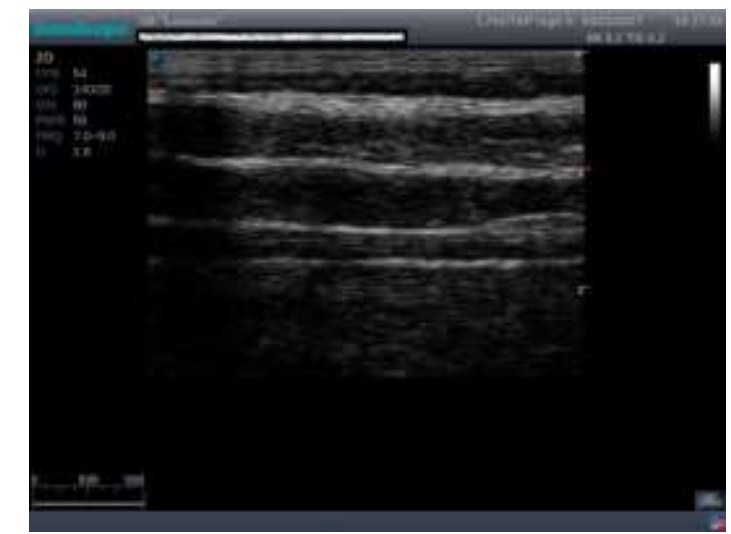

Figure 1. Ultrasound image of the skin, subcutaneous tissue, fat and the three abdominal wall muscles (external and internal oblique abdominal muscle and transverse abdominal muscle), including the peritoneum.

With a $22 \mathrm{G}$ needle (Stimuplex D Plus $22 \mathrm{G} \times$ $80 \mathrm{~mm}$ ), under real-time ultrasound visual control, $20 \mathrm{ml}$ of $0.375 \%$ ropivacaine was injected bilaterally with intermittent aspiration. The injection was between the aponeurosis of the internal oblique and transversus abdominis muscles. The correct placement of the local anesthetic was identified ultrasonographically by a dark shadow, the "stratification" of the aponeurosis between the inner oblique and the transversal muscle (Figure 2). Patients were under observation in the recovery room for one hour after the extubation.

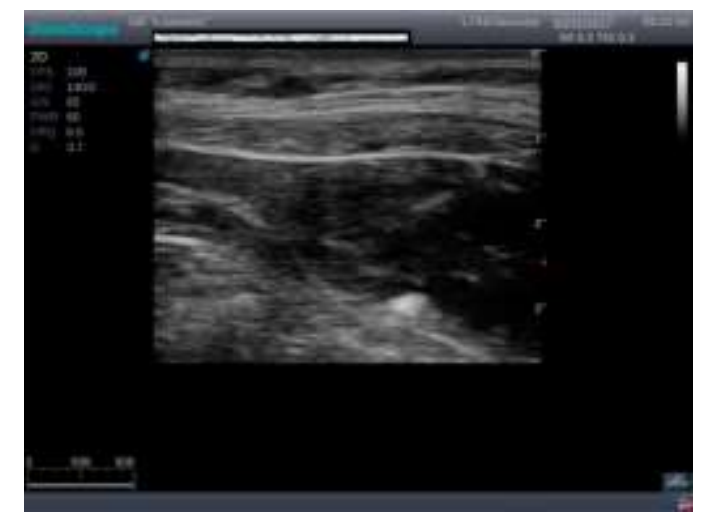

Figure 2. Ultrasound image of correct placement of the local anesthetic (dark shadow) in the aponeurosis between the inner oblique and the transversal muscle
The degree of pain was assessed using a visual-analog 10-point scale, VAS (0: no pain, 10: intolerable pain) $[13,14]$. The pain was also evaluated in the postoperative period of the 1st, 2nd, 6th, 12th and 24th hours (purpose of another study). We administered NSAIDs Paracetamol $1000 \mathrm{mg}$, in combination with Ketoprofen $100 \mathrm{mg}$, only in patients with VAS score above 5 .

Neither complications were detected, nor did adverse reactions of the opioid analgesic, Fentanyl in both patient groups.

The data obtained were presented as mean \pm standard deviation (Mean \pm SD). Statistical processing and correlation analysis of the experimental data in Microsoft Excel, ver. 2010, a student's t-test was used to determine statistical significance. Statistical significance was defined at $\mathrm{p}<0.05$ (significance level $\alpha>$ 95\%).

\section{RESULTS}

The average anesthesia time was 151 minutes, while the estimated surgery time was 132 minutes in both groups. Significant time differences between both groups were not detected.

We have monitored intraoperatively in the interval of 10 minutes: heart rate, non-invasive blood pressure, electrocardiogram, pulse oximetry (SpO2), bispectral index (BIS). Significant differences in pulse oximetry, capnography and BIS index were not found.

An analysis of the intraoperative results of the hemodynamic parameters revealed that the mean heart rate, systolic and diastolic blood pressure, immediately after the surgical incision of the skin, were significantly higher in both groups, which led to additional administration of Fentanyl.

The study results showed tachycardia, between the 45th and 120th minutes of the surgical procedure, and after extubation, in patients in group I (GA) compared to group II (GA + TAP). Systolic and diastolic pressures during this time period (T6 - T9) were also significantly higher, hypertension, in the control group of patients as opposed to experimental group. Intraoperative hemodynamic changes are presented in Figures 3 and 4. 


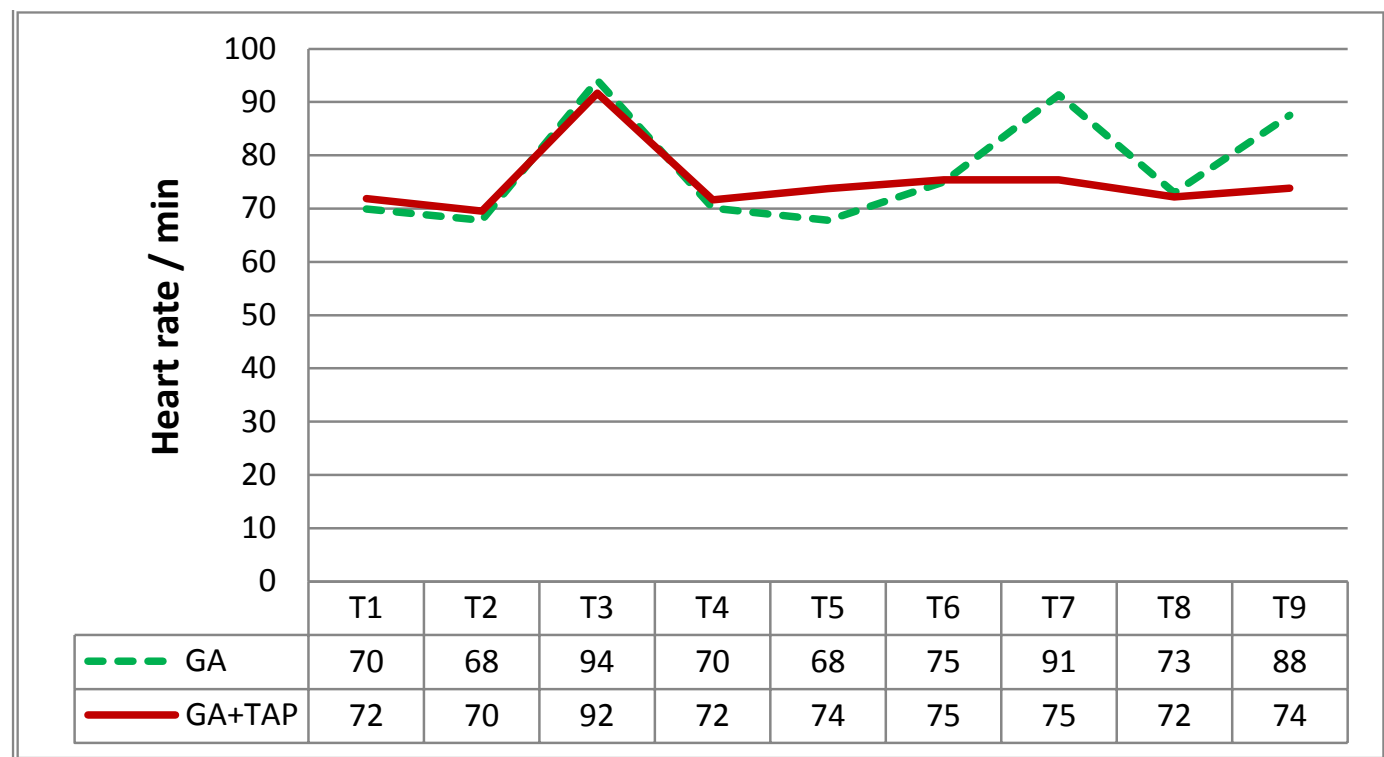

Figure 3. Comparison of heart rate between control (GA) and experimental groups (GA + TAP) at different time interval: T1 - before anesthesia; T2 - after anesthesia; T3 - after surgical incision of the skin; T4 - 15 min; T5 30 min.; T6 - 45 minutes; T7 - 60 min.; T8 - 120 min .; T9 - after extubation.

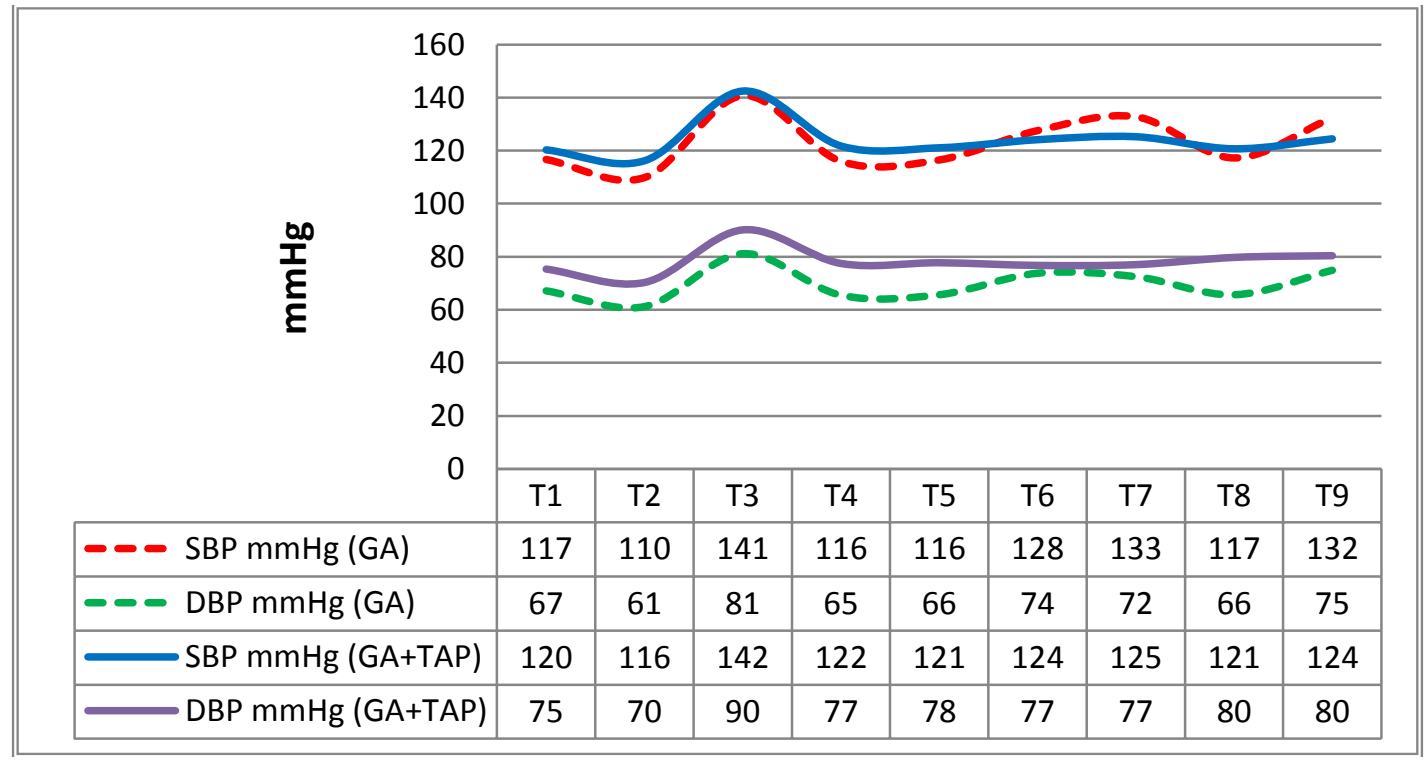

Figure 4. Comparison of systolic and diastolic blood pressure between control (GA) and experimental groups (GA + TAP) at different time interval: T1 - prior to anesthesia; T2 - after anesthesia; T3 - after surgical incision of the skin; T4 - 15 min; T5 - 30 min .; T6 - 45 minutes; T7 - 60 min .; T8 - 120 min .; T9 - after extubation.

Total amount of Fentanyl used intraoperatively for induction of anesthesia, skin incision and during surgery for the two groups of patients: GA - general anesthesia without TAP block (control group) and GA+TAP - general anesthesia and TAP block (experimental group) was shown in Table 2. The total amount of Fentanyl was significantly less in the patients in the experimental group $(\mathrm{GA}+$ TAP).

Table 2. The amount of fentanyl used intraoperatively. Data are presented as (Mean \pm SD).

\begin{tabular}{||l|l|l|l|l||}
\hline \multicolumn{5}{|c|}{ The amount used of fentanyl, $\boldsymbol{\mu g}$} \\
\hline Group & $\begin{array}{l}\text { Induction of } \\
\text { GA }\end{array}$ & Skin incision & During surgery & Total \\
\hline GA & $\mathbf{5 2 , 9} \pm 12,127$ & $\mathbf{5 7 , 4} \pm 17,15$ & $\mathbf{5 8 , 8} \pm 19,648$ & $\mathbf{1 6 9 , 1} \pm 25,814$ \\
\hline GA+TAP & $\mathbf{5 1 , 4} \pm 8,45$ & $\mathbf{3 5 , 7} \pm 28,62$ & $\mathbf{2 7 , 1} \pm 30,54$ & $\mathbf{1 0 9 , 7} \pm 32,24$ \\
\hline
\end{tabular}


The results for postoperative pain, assessed on a visual-analogue scale (VAS), were obtained after extubation and when patients were moved from the operating desk to the hospital bed are shown in Table 3.

Table 3. Results of the Visual Analog Scale (VAS), VAS score.

\begin{tabular}{|c|c|c|c|}
\hline \multirow{2}{*}{ Reporting time } & \multicolumn{2}{|c|}{ Pain score, VAS score (Mean \pm SD) } & Significance parameter value $\boldsymbol{p}$ \\
\cline { 2 - 4 } & GA & GA + TAP & \\
\hline After extubation & $4,2 \pm 1,39$ & $1,4 \pm 0,86$ & $3.10^{-8}$ \\
\hline Transfer * & $5,1 \pm 0,83$ & $2,1 \pm 1,1$ & $2.10^{-10}$ \\
\hline
\end{tabular}

*Transfer - patients were moved from the operating desk to the hospital bed

The results of Table $\mathbf{3}$, which are also presented graphically in Figure 5, showed significantly lower degree of pain immediately after surgery in patients in the experimental group.

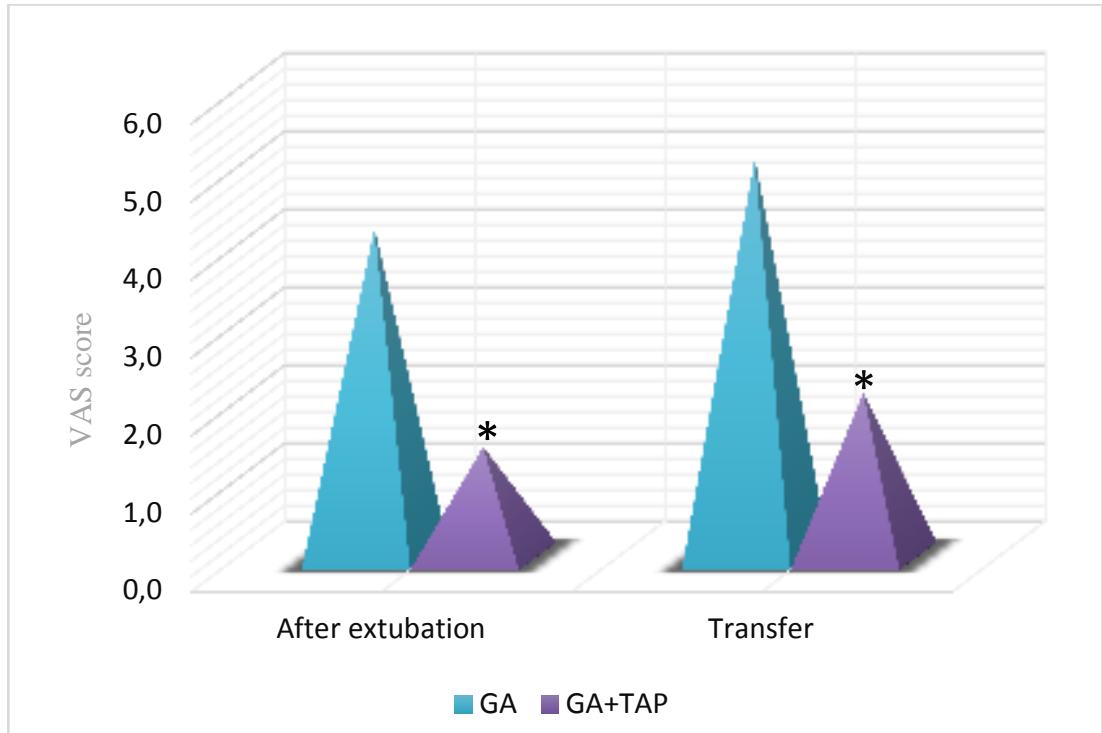

Figure 5. Results of the pain VAS score, in the control (GA) and experimental (GA + TAP block) group. ${ }^{*} \mathrm{p}$ $<0,05$ - significant difference between the results of the two groups: GA and GA + TAP

\section{CONCLUSION}

Ultrasound-guided bilateral peripheral block (TAP - block) with $0,375 \%$ ropivacaine, administered to patients undergoing bilateral laparoscopic inguinal repair, significant reduces intraoperative pain symptoms; opioid analgesic consumption and VAS score in the first postoperative hour. TAP - block is part of the multimodal anesthesia and adjuvant for intraoperative and post-operative analgesia. Further research is essential to establish the optimum use of this technique.

\section{REFERENCES}

1. K. Gritsenko, Y. Khelemsky, A. David Kaye, N. Vadivelu, R. Urman, "Multimodal therapy in perioperative analgesia", Best Practice \& Research Clinical Anaesthesiology, doi: 10.1016/j.bpa.2014.03.001,

2014 , [PubMed] [Google Scholar]

2. De Oliveira GS Jr, Castro-Alves LJ, Nader A, Kendall MC, McCarthy RJ. Transversus abdominis plane block to ameliorate postoperative pain outcomes after laparoscopic surgery: a meta-analysis of randomized controlled trials. Anesth Analg 2014;118(2):454-

463 [Crossref] [Google Scholar]

3. Venkatraman, R., Abhinaya, R. J., Sakthivel, A., Sivarajan, G., "Efficacy of ultrasound-guided transversus abdominis plane block for postoperative analgesia in patients undergoing inguinal hernia repair", Local and Regional Anesthesia, 18(9), 7 12, 2016.

4. Rafi AN. "Abdominal field block: A new approach via the lumbar triangle", 
Anaesthesia,

6. [PubMed] [Google Scholar]

5. Hebbard P., Y. Fujiwara, Y. Shibata, et al. Ultrasound-guidedtransversus abdominis plane (TAP) block, Anaesth Intensive Care, 2007, 35, 616-617.

6. M. Gajanan Mankikar, S. Pravin Sardesai, P. Sachin Ghodki, "Ultrasound-guided transversus abdominis plane block for postoperative analgesia in patients undergoing caesarean section", J Anaesth 2016;60:2537, 2016[PubMed] [Google Scholar]

7. McDonnell JG, Curley G, Carney J, Benton A, Costello J, Maharaj $\mathrm{CH}$, et al. "The analgesic efficacy of transversus abdominis plane block after cesarean delivery: A randomized controlled trial", Anesth Analg. 2008;106:186-

91.[PubMed] [Google Scholar]

8. Milone, M., Di Minno, M. N., Musella, M., Maietta, P., Salvatore, G., Lacovazzo, C. et al., "Outpatient inguinal hernia repair under local anaesthesia: feasibility and efficacy of ultrasound-guided transversus abdominis plane block", Hernia, 17(6), 749-755, 2013. [PubMed] [Google Scholar]

9. Takebayashi, K., Matsumura, M., Kawai, Y., Hoashi, T., Katsura, N., Fukuda, S., Shimizu, K., Inada, T., Sato, M., "Efficacy of Transversus Abdominis Plane Block and Rectus Sheath Block in Laparoscopic Inguinal Hernia Surger", Int Surg, 100(4), 666-671, 2015. [PubMed] [Google Scholar]
10.Niraj G, Searle A, Mathews M, et al. Analgesic efficacy of ultrasound-guided transversus abdominis plane block in patients undergoing open appendectomy. $\mathrm{Br}$ J Anaesth. 2009. doi:10.1093/bja/aep175[PubMed] [Google Scholar]

11.C. J. Walter, C.MaxwellArmstrong,T.D. Pinkney, P. J. Conaghan,N.Bedforth, C. B. Gornall, A. G. Acheson. A randomised controlled trial of the efficacy of ultrasound-guided transversus abdominis plane (TAP) block in laparoscopic colorectal surgery, Surgical endoscopy, Vol.27, 2366-2372, 2013 [PubMed] [Google Scholar]

12.McDonnell JG, O’Donnell B, Curley G, Heffernan A, Power C, Laffey JG. The analgesic efficacy of transversus abdominis plane block after abdominal surgery: a prospective randomized controlled trial. Anesth Analg 2007;104:193-7

13.O'Donnell BD, McDonnell JG, McShane AJ. The transversus abdominis plane (TAP) block in open retropubic prostatectomy. Reg Anesth Pain Med 2006;31:91

14.Hawker G. A., S. Mian, T. Kendzerska, M. French, Measures of Adult Pain, Arthritis Care \& Research, 63(S11), 2011, S240S252. 